\title{
Analysis of Plant Height Measuring Method Based on Line Laser Yangyang $\mathrm{Li}^{1, \mathrm{a}}$, Ping Song ${ }^{1, \mathrm{~b}}$ and Yayu Zhai ${ }^{1, \mathrm{c}}$ \\ ${ }^{1}$ Key Laboratory of Biomimetic Robots and Systems, Beijing Institute of Technology, Beijing 100081 , China;

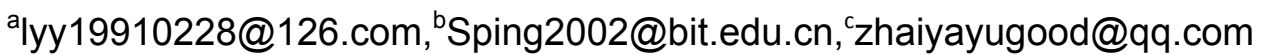

Keywords:Line laser, error, ranging.

\begin{abstract}
Laser has the characteristics of good monochromaticity, excellent coherence, well directivity and high brightness. With the features of non-touching, not easy to damage the surface, wide adaptability of materials, simple structure, strong anti-interference ability, and measuring fast, Laser ranging is widely used in the field of geometric measurement. In this paper, the height of the plantis calculated from the picture captured from a camera with the assist of line laser, and the parameters that may affect the height measurement accuracy are analyzed. Finally, the feasibility about how to choose the parameters is presented which will do some help for measuring.
\end{abstract}

\section{Introduction}

The biomass of grassland is the production of grass of certain area in grassland. The measurement and estimation of the biomass of grassland not only have important significance on the study of the vegetation production, carbon cycling and nutrient distribution of terrestrial ecosystem, but also affect the measurement of other biological parameters, which will influence how people will develop and utilize the surface vegetation[1]. Vegetation height is an important parameter of biomass, and it plays an important role in the accurate measurement of edible biomass of grassland [2].

Nowadays, there are various vegetation height measuring methods such as using Ruler, ultrasonic sensor or TOF camera etc. The device of using ruler or ultrasonic is simple but the accuracy is low. Using TOF camera to measure is of high accuracy but it costs a lot. This paper introduces a kind of new method to measure the height of plant with line laser and ordinary camera, which is both of high accuracy and low cost. The system will capture the laser line on the plant with the camera firstly, and then separate the laser line out through some image processing algorithm. At last we calculate the height of laser line and analyze the impact factors which will provide some help for measuring platform construction.

\section{Architecture of The Height Measuring System Based on Line Laser}

The 3D line laser measuring system consists of a laser emitting device, an image acquisition device, a steering engine and a control deviceas shown in Fig.1. The laser emitting devicewill emit a laser line illuminating the plant, and then the image acquisition device will capture the image and calculate the height of laser line through the control device. The steering engine can rotate a certain angle so that all the plant can be scanned. There are also some other 3D scanning ways, like in [3] where the scanner moves straight. 


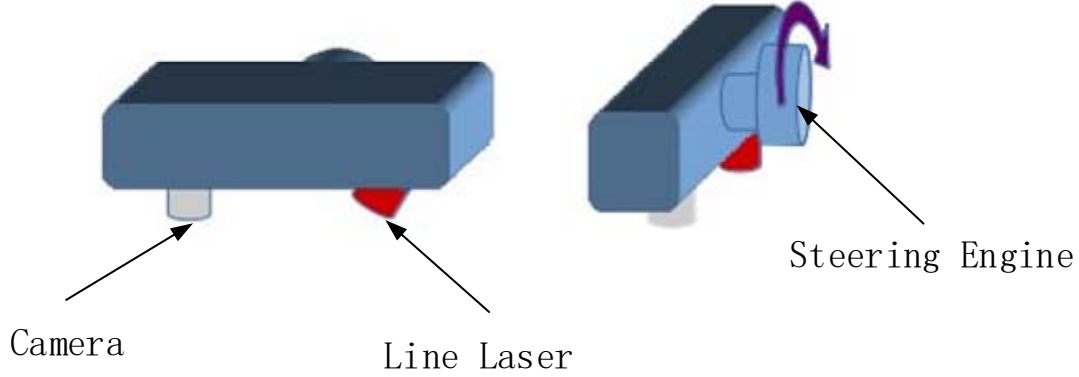

Fig.13D line laser measuring system

\section{The Height Measuring Principle}

The principle of line laser ranging is similar to that of the single-point laser ranging principle, which is based on the principle of triangulation as shown in Fig. 2[4,5]. The distance between the line laser and ground is $H$, and the included angle between line laser and baseline is $\beta$. $L$ stands for the distance between line laser and camera. $X$ represents the pixel distance between the object and the optical axis on the imaging plane. The distance between the line laser and the object is $h$ and PixelSizestands for the single pixel unit size. Based on the similar principle of triangle $\triangle \mathrm{OAB} \backsim \triangle \mathrm{DCO}$, we can come the conclusion:

$$
\frac{D C}{f}=\frac{O A}{h}
$$

So we know that:

$$
h=f * L /\left(\frac{f}{\tan \beta}+X * \text { PixelSize }\right)
$$

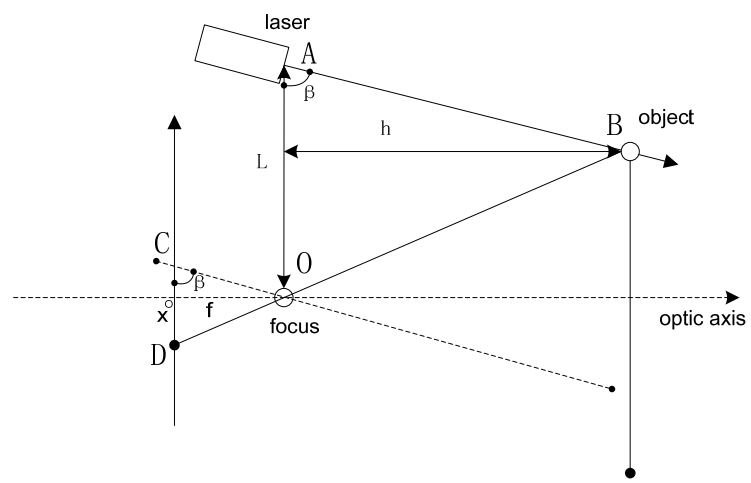

Fig.2 The object images below the optical axis

When the object images above the optical axis as shown in Fig. 3, we can still come to the conclusion that

$$
h=f^{*} L /\left(\frac{f}{\tan \beta}-X^{*} \text { PixelSize }\right)
$$




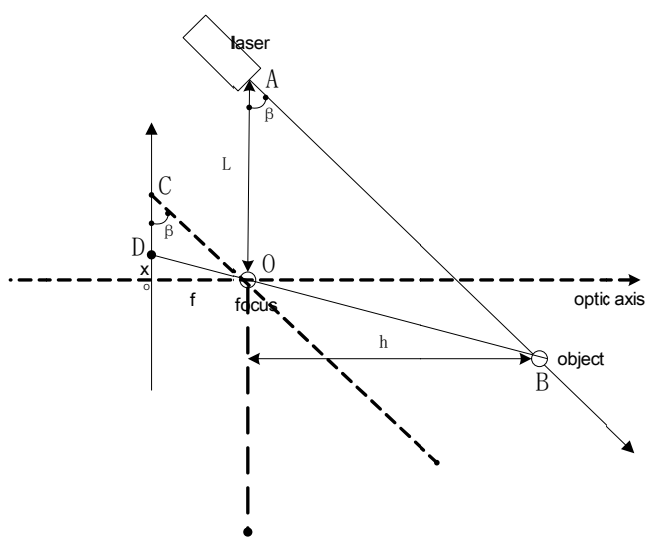

Fig. 3 The object images above the optical axis

After we calculate the length of $h$, if the distance between the line laser and the ground $H$ is known, it's easy to know that the height of plant $h$ '.

$$
h^{\prime}=H-h(4)
$$

\section{Extraction of The Laser Line and Ranging Analysis}

Based on the laser ranging principle, we capture a vertical image sample as shown in Fig. 4.The image size camera captured is $3464 * 2448$, and the camera focal length is $4.12 \mathrm{~mm}$. The length of baseline called $\mathrm{L}$ is as long as $340 \mathrm{~mm}$, and the included angle $\beta$ is $54^{\circ}$. The line laser is $820 \mathrm{~mm}$ away from the ground. According to the color difference of RGB, a binarization process is made as shown in the Fig. 5[6]. As a result, every single-point's coordinate information on the laser line is got. When the abscissa of the pixel point on the laser line is less than 1224 , which means the imaging position is below the optical axis, we can get the range information through the formula (1).When the abscissa of the pixel point on the laser line is greater than 1224 , which means the imaging position is above the optical axis, we can get the range information through the formula (2). As shown in Figure 6, we set the ordinate of the laser spot as abscissa, and the height of the plant as ordinate. Since there exist height differences of plant, some of the laser line can't be captured. For these points shown in bold in Fig. 6, we set the nearest laser point that captured as their height[7].

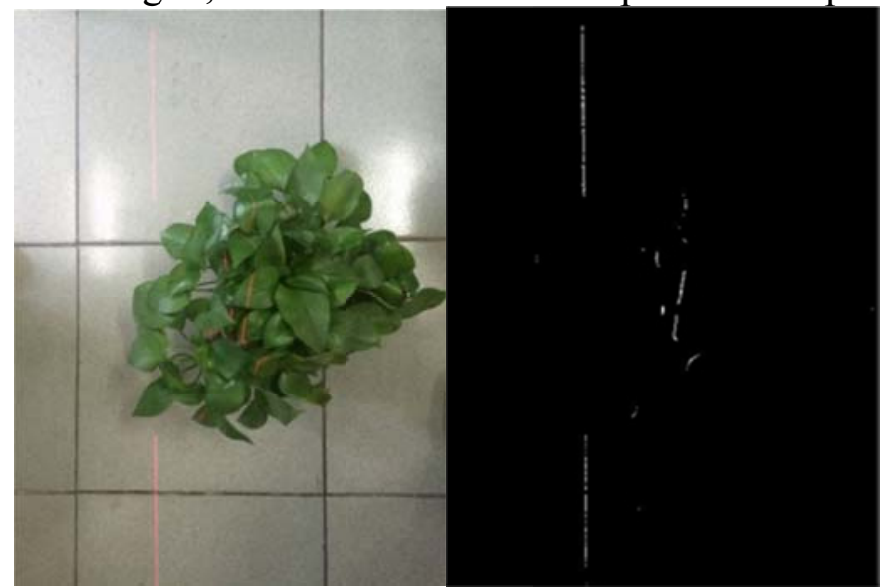

Fig.4 A vertical captured image with laser lineFig.5Binarization image 


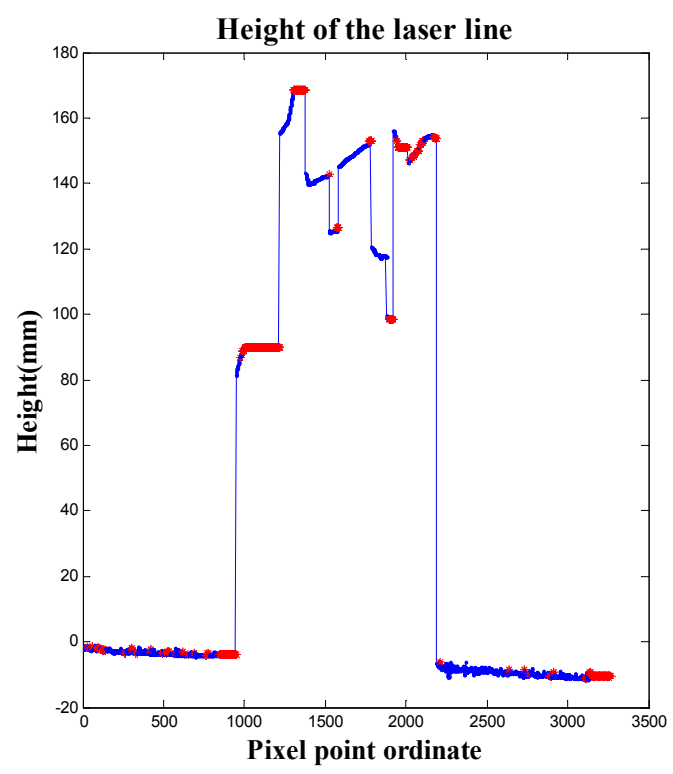

Fig.6 Height of laser line

\section{Analysis of Errors}

According to the height calculation equation (2) and (3), it's easy to see that the main factors that affect the ranging are baseline $L$, focal length $f$, the included angle between laser and baseline $\beta$. Since the focal length of camera is fixed, the height is highly influenced by $L$ and $\beta$. Now we are going to discuss about how strong influence $L$ and $\beta$ will cause, when the object images below the optical axis. And it's similar when the object images above the optical axis.

(1) When $L$ is fixed, we calculate the $h$ derivation of $X$.

$$
\frac{d h}{d X}=\frac{-f L^{*} \text { PixelSize }}{\left(\frac{f}{\tan \beta}+X^{*} \text { PixelSize }\right)^{2}}
$$

From the equation(5) we can see that the further the distance, the bigger the error of ranging. In the Fig. 7, we make a small difference on $\beta$ and draw two height lines from which we can also get the conclusion[8].

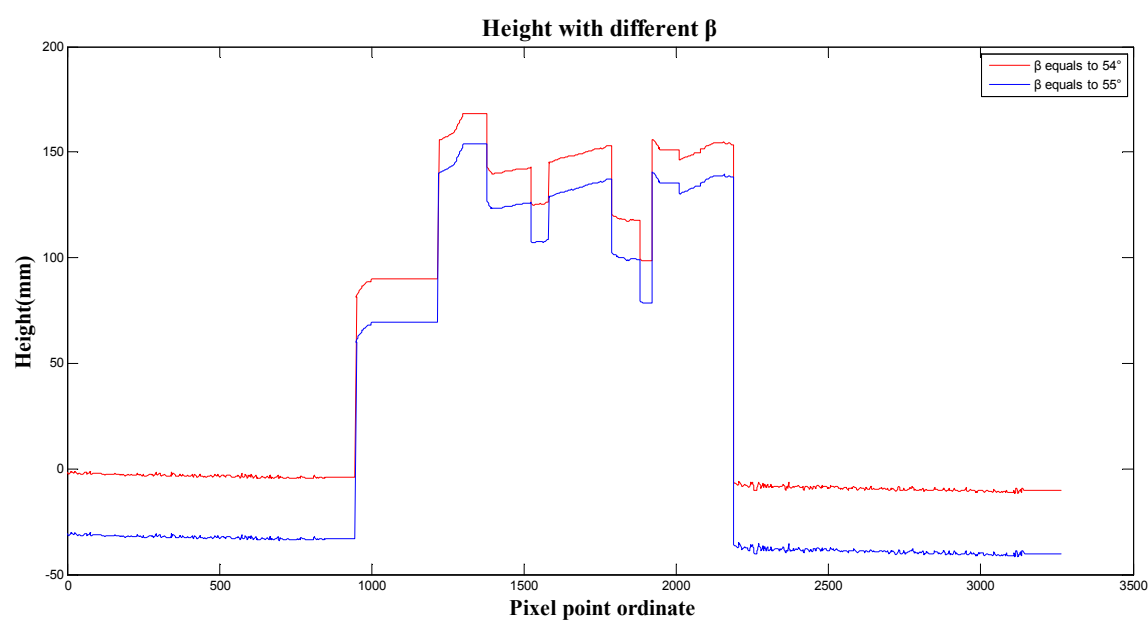

Fig. 7 Height of laser line when $\beta$ equals to $54^{\circ}$ and $55^{\circ}$

(2) For a certain pixel coordinate point $(1116,1632)$, we calculate the $h$ derivation of $\beta$.

$$
\frac{d h}{d \beta}=\frac{f L * \sec ^{2} \beta}{\left(f+X * \text { PixelSize } * \tan ^{2} \beta\right)^{2}}
$$

We make a height curve about the $\beta$ anglewhose deviation is positive twenty degrees. And we can 
see that the deviation of $\beta$ has great influence on the ranging as shown in Fig. 8. In order to reduce the influence of $\beta$ and make the value of $\mathrm{X}$ matter most, $\beta$ 's value should be as large as possible, but it can't be infinity. According to the equation (7), we can work out a maximum of $\beta$ referring to the range of $h$ and length of $L$.

$$
\frac{f}{\tan \beta}=\frac{f L}{h}+X^{*} \text { PixelSize }
$$

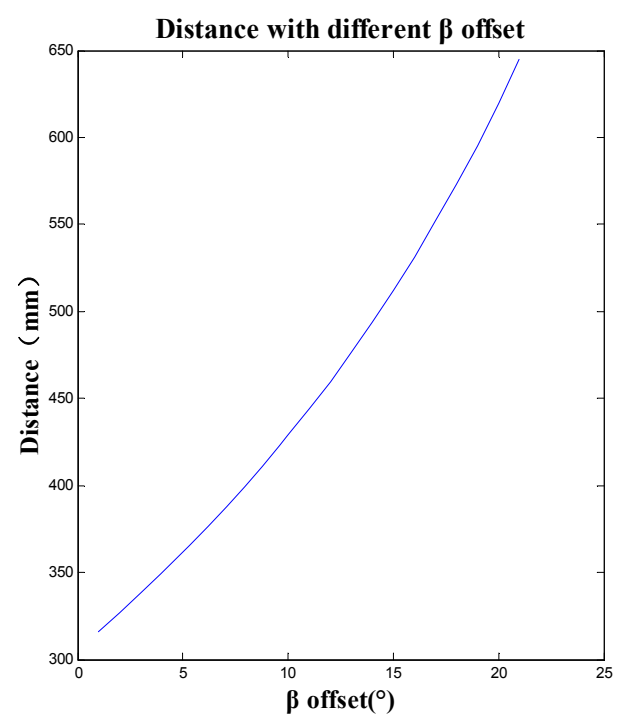

Fig. 8 ranging with different $\beta$ for a certain point

(3) If there is no error of $\beta$, for a certain point $(1116,1632)$, we calculate the $h$ derivation of $L$.

$$
\frac{d h}{d L}=\frac{f^{*} \text { PixelSize }}{\left(\frac{f}{\tan \beta}+X^{*} \text { PixelSize }\right)^{2}}
$$

It's easy to get the result that the deviation of $L$ and the deviation of distance $h$ are linear correlation.

The errors got from $L$ and $\beta$ are system errors, while $\beta$ has greater impact on the ranging. We can reduce them as much as possible, but there also exists some other errors.In the process of capturing image, there is an assumption that we treat our camera as a pinhole camera model. In reality, the camera is usually focused on optical lenses to image, besides the lenses' surface is not parabolic, and the light-sensitive CMOS chip is not strictly parallel to the lens so that there will be some distortion on the image we captured. Meanwhile, if the camera's optical axis is not in the middle of the image, it will affect the calculation of the value of $X$, which will also cause some errors[9].

\section{Conclusions}

Through the analysis of the different parameters, we can get the conclusion that angle $\beta$ has the greatest effect on the height measurement. Comparing with angle $\beta$, the length of baseline and camera parametershave less impact. When the system equipment being installed, considering the size of the device, we can calculate a best angle $\beta$ referring to the formula (7), so that the system errors can be minimized and meet the required precision, which can offer some constructive advice for plant height measuring.

\section{References}

[1]Tilman D, Wedin D, Knops J. Productivity and sustainability influenced by biodiversity in grassland ecosystems[J]. Nature, 1996, 379(6567): 718-720. 
[2] Bonham C D. Measurements for terrestrial vegetation[M]. John Wiley \& Sons, 2013.

[3] Xi F, Liu Y, Feng H Y. Error compensation for three-dimensional line laser scanning data[J]. The International Journal of Advanced Manufacturing Technology, 2001, 18(3): 211-216.

[4] K. Konolige, J. Augenbraun, N. Donaldson, C. Fiebig, and P. Shah. A low-cost laser distance sensor. In Int. Conference on Robotics and Automation (ICRA), 2008.

[5]GuichunC.Laser Triangle Method for Measuring Microdisplacement [J]. TOOL ENGINEERING, 1997, 6: 014.

[6] Camera Calibration Toolbox for Matlab®, http: //www. vision. caltech. Edu /bouguetj/calib_doc /index. html .

[7] Fisher, R. B. and D. K. Naidu. A Comparison of Algorithms for Subpixel Peak Detection.Springer-Verlag, Heidelberg, 1996.

[8]Self-made low cost 3D laser scanning range finder: www.csksoft.net /blog/post/1 owcost 3d laser_ranger_1.html.

[9] Mertz, C., J. Kozar, J.R. Miller, and C. Thorpe. Eye-safe Laser Line Striper for Outside Use. Intelligent Vehicle Symposium, 2002. 